\section{THEMATIC} PAPER Head of Liaison Psychiatry Unit,
Associate Professor, Department Associate Professor, Depa
of Psychiatry, Pontificia Universidad Católica de Chile, Santiago, Chile, email jcaldep@ gmail.com

\title{
Stigma in Latin America
}

\author{
Jorge Calderon
}

Despite the fact there has been difficulty in developing a theory of stigma that is specific to patients with mental disorders, a multidisciplinary effort has been made to understand its impact (Smith, 2002). The key element in the social origins of stigma is the perception of difference, in particular societal concerns that people with mental disorders, who often have communication problems, harbour undesirable traits such as potential violence and other unpredictable behaviours (Byrne, 2000). In order for us to overcome barriers to the implementation of effective stigma-reduction programmes, we need to understand more about the public's misperceptions of mental illness, including the origins of prejudice and discrimination.

In industrialised societies, the impact of education and the media has led in recent years to an increasingly widespread understanding of mental illness as a medical problem. However, it is unfortunate that despite this enlightenment, many in society continue to be suspicious of, and even rejecting of, people with mental illness. Consequently, educational campaigns directed at ensuring their inclusion, which seek to influence wider cultural contexts, become relevant (Pescosolido et al, 2013). To date, most research into stigma and mental illness has been carried out in North America and Europe and has focused upon schizophrenia.

In much of Latin America there has been a widely implemented switch away from an asylum model of psychiatric care to community-based services in recent years. In a number of countries, that change has been accompanied by new mental health legislation, which has centred on patients' rights. In this issue of BJPsych International, we present a series of papers that reflect on the origins and management of stigma towards psychiatric patients in the region. Acuna et al inform us that in Latin America and the Caribbean, mental and neurological disorders account between them for over $20 \%$ of the total burden of disease, but resources are scarce and the treatment gap between resources for mental and physical disorders in many countries is substantial. Different ways of reducing this gap and fighting stigma are suggested.

The adoption of resolutions regarding universal access to health and universal health coverage by members of the Pan American Health Organization may help to increase the provision of resources for psychiatric care and encourage its integration with primary healthcare. As described by Dahl et $a l$ in their report from Brazil, novel interventions such as peer support groups implemented by peer support workers and community mental health groups may help to modify patients' narratives about their illness and overcome stigma.

Finally, Agrest and colleagues describe a corpus of new studies on stigma which is beginning to shed light on the similarities and differences between the characteristics of societal stigma towards people with mental illness in Argentina and those found in North America and Europe.

\section{References}

Byrne, P. (2000) Stigma of mental illness and ways of diminishing it. Advances in Psychiatric Treatment, 6, 65-72.

Pescosolido, B. A., Medina, T. R., Martin, J. K., et al (2013) The 'backbone' of stigma: identifying the global core of public prejudice associated with mental illness. American Journal of Public Health, 103, 853-860.

Smith, M. (2002) Stigma. Advances in Psychiatric Treatment, 6 , 317-323.

\section{THEMATIC} PAPER

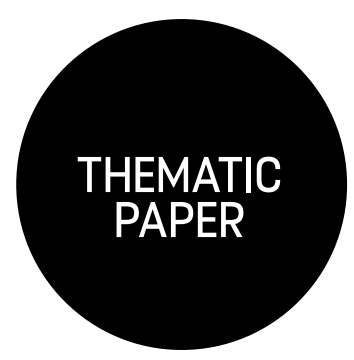

\section{Stigma and psychiatric care in Latin America: its inclusion on the universal health coverage agenda}

\author{
Cecilia Acuña, ${ }^{1}$ Rafael Sepúlveda² and Osvaldo Salgado ${ }^{3}$
}

${ }^{1}$ Advisor, Health Systems and Services, Pan American Health Organization/World Health Organization, (unization, Ecuador, email acunamar@paho.org ${ }^{2}$ Chief, Mental Health

Department, Ministry of Health, Chile

${ }^{3}$ Director, South Metropolitan Health Area, Ministry of Health, Chile
In 2014, the Pan American Health Organization (PAHO) approved Resolution CP53.R14, which aimed to provide a framework for universal access to health and universal health coverage. It sets the stage for the inclusion of psychiatric practice within the provision of universal healthcare and highlights the fight against stigma. We propose to concentrate our efforts on changing the model of medical management. To that end, we are promoting the inclusion of mental health patients within the daily routine of primary care centres, thus allowing them to interact with other users of health services on a regular basis. 


\section{Background}

One of the key aims of Resolution CD53.R14 ('Strategy for Universal Access to Health and Universal Health Coverage'), which was agreed in September 2014 by all member states at the 53rd Directive Council of the Pan American Health Organization (PAHO), concerns the provision of universal access to people-centred health services in the Americas region. The resolution urges participating countries to move towards providing universal access to comprehensive, high-quality and progressively expanded health services that are consistent with broad population health needs, system capacities and the national context. It emphasises that there are unmet and undifferentiated population requirements from such a system, as well as specific needs of groups who are particularly vulnerable. The resolution recognises the importance of defining and implementing actions to improve the organisation and management of health services through the development of healthcare models that focus on the diverse needs of people and communities. There is an unfulfilled demand to improve response capacity at the primary level of care, through the development of integrated health services networks.

At that same meeting, PAHO member states approved Resolution CD53.8, 'Plan of Action on Mental Health'. Based on information gathered from the World Health Organization's Assessment Instrument for Mental Health Systems (WHO-AIMS) (PAHO, 2013), the Plan of Action's background document reports that in Latin America and the Caribbean, mental and neurological disorders account for $22.2 \%$ of the total burden of disease, measured in disabilityadjusted life-years (DALYs). Resources allocated by countries to tackle the burden of disease related to mental health problems are insufficient and inequitably distributed. This treatment gap in the provision of resources is, in many countries, more than $70 \%$. Although the document acknowledges that there are key factors that account for the relative neglect of mental health as a priority for investment - including stigma, social exclusion and discrimination against patients with psychiatric conditions - no specific actions to tackle stigma are included in the Plan of Action.

The World Health Organization (WHO, 2013) has highlighted the widespread human rights violations and discrimination experienced by people with mental disorders. It calls for a human rights perspective to respond to the global burden of mental disorders. Its Mental Health Action Plan 2013-2020 proposes that the formulation of strategies for mental health promotion, with the aim of preventing mental disorders across the life course, should focus on antidiscrimination laws and should encourage information campaigns (WHO, 2013). There should be an effort to redress the stigmatisation and human rights violations associated with mental disorders. The Action Plan requests the WHO secretariat to provide technical support to assist in the implementation of evidence-based best practices for reducing stigmatisation and discrimination, and to promote human rights, but it does not identify the best practices by which these goals might be achieved. This is an important omission.

\section{Positioning psychiatric care and stigma on the universal health coverage agenda}

The concept of universal health coverage received strong support in the unanimous adoption of Resolution 67/L.36 'Global Health and Foreign Policy' by the United Nations General Assembly in December 2012. It urges governments to move towards ensuring all people fall within the healthcare framework and to work on the determinants of better healthcare provision within the sectors of mental and physical disorder. It thus sets a political agenda for positioning psychiatric care within universal health coverage, and encourages the development of strategies to eliminate stigma as a barrier that prevents patients from accessing the care they need. Accordingly, it is now of critical importance to identify the best strategies by which to implement this resolution.

At the 29th Congress of the International Academy of Rights and Mental Health (Paris, July 2005), Acuña \& Bolis proposed specific strategies to ensure that stigma is included on the political and health agenda. These include:

- highlighting the role that stigma plays as a barrier, impeding access to healthcare

- providing information to people with mental health problems about the facilities available to them, and advocating for the rights of people with mental illness

- educating and training health workers at the primary care level

- encouraging community leaders to address the health needs of people with mental illness

- strengthening legal mechanisms so that people with mental health difficulties can exercise their rights, especially in relation to healthcare.

Some of these strategies are also included in the PAHO's Plan of Action on Mental Health, as well as in the WHO's Mental Health Action Plan 2013-2020.

\section{Changing the model of care}

So that we can make progress on the goal of eliminating stigma as a barrier to access, best practices and specific actions must be identified. In addition to those ideas proposed by Acuña \& Bolis, other strategies also could be adopted. For example, it is now widely accepted that communitybased models of care, with a focus on promoting recovery, should replace psychiatric hospitals and allow the social reintegration of patients, as suggested by PAHO Resolution CD53.R14. Our own proposal is based on the work of Lopez et al (2008); we would like to see the exposure of mental health patients to other users of health services at the primary care level, and the inclusion of psychiatric patients in the day-to-day routine of their 
community health centres. If this idea is implemented, other patients and users of health services will have the opportunity to meet and interact with people who have psychiatric disorders. By these means we hope to reduce the prejudicial attitudes that stigmatise people who are psychiatrically ill as dangerous and threatening. It will help to ensure they are regarded in future simply as members of the community, just like everyone else.

\section{Conclusion}

The adoption of resolutions regarding universal access to health and universal health coverage by PAHO and WHO member states as well as by the United Nations General Assembly provides a unique window of opportunity for us to promote the universality of psychiatric care. It can assist in our fight against the stigma that psychiatric patients currently experience in so many regions of the world. But to eliminate stigma, we need to identify and to implement specific evidence-based strategies. Changing the model of medical care, with a focus on integrating psychiatric care into the everyday practice of primary healthcare and promoting exposure of psychiatric patients to other users of health services, should be a centrepiece of those strategies.

\section{References}

López, M., Laviana, M., Fernández, L., et al (2008) La lucha contra el estigma y la discriminación en salud mental. Una estrategia compleja basada en la información disponible. [The fight against stigma and discrimination in mental health. A complex strategy based on the available information.] Journal of the Spanish Association of Neuropsychiatry, 28, 43-83.

PAHO (2013) WHO-AIMS: Report on Mental Health Systems in Latin America and the Caribbean. Pan American Health Organization.

WHO (2013) Mental Health Action Plan 2013-2020. World Health Organization.

\section{THEMATIC PAPER}

\title{
Stigma and recovery in the narratives of peer support workers in Rio de Janeiro, Brazil
}

\author{
Catarina Magalhães Dahl, ${ }^{1}$ Flavia Mitkiewicz de Souza, ${ }^{2}$ Giovanni Marcos \\ Lovisi ${ }^{3}$ and Maria Tavares Cavalcanti ${ }^{4}$
}

'Psychologist and PhD Student, Institute of Psychiatry, Federal University of Rio de Janeiro, Brazil, email catdah|@hotmail. com

2Psychologist and PhD Student, Institute of Psychiatry, Federal University of Rio de Janeiro, Brazil

${ }^{3}$ Associate Professor of Collective Health, Institute of Collective

Health Studies, Federal University Rio de Janeiro, Brazil

${ }^{4}$ Associate Professor of Psychiatry and Director, Institute of

Psychiatry, Federal University of Rio de Janeiro, Brazil

Acknowledgements: We would like to acknowledge the National Institute of Mental Health (NIMH grant no. 1 U19 MH095718. 01) and the National Council for Scientific and Technological Development (CNPq grant no. 470217/2012-0) for financial support. We also wish to acknowledge all RedeAmericas' partners and, last but not least, partners and, last but not leas the peer support workers and mental health users who directly or indirectly contributed to this work.
Stigma attaching to mental illness has been considered a major challenge to public policies, to the provision of care and to the well-being of people who live with the experience of mental illness worldwide. Here we discuss narratives from peer support workers which we obtained during the assessment of a new psychosocial intervention programme in Rio de Janeiro. We used a range of focus groups, in-depth interviews and clinical supervision notes to derive these narratives, which covered topics such as the peer support workers' perceptions of family and social views, their sense of self and the experience of being stigmatised. We conclude that stigma appears to be a barrier to recovery. Peer support work for people with severe mental illness is a strategy that may help them to overcome stigma and discrimination. Fostering mutuality and hope in the context of peer support helps affected individuals to make sense of their being in the world and can facilitate their recovery.

\section{Background}

Stigma presents a challenge to public policies, to the provision of care and to persons worldwide who live with the experience of mental illness (Saraceno et al, 2007; Sartorius, 2007). Characterised by negative thoughts and attitudes, stigma results from lack of knowledge (ignorance), prejudicial attitudes and discriminatory behaviour (Thornicroft et al, 2007). The experience of stigma decreases self-esteem, which in turn reinforces negative attitudes and increases the risk of social exclusion (Sartorius, 2007). Stigmatisation is a devastating experience that negatively impacts upon self-identity and creates a barrier to recovery, to accessing health services, to getting a job and to education and housing. Whereas an important step to recovery is accepting one's illness, on the other hand, the label of a psychiatric diagnosis can lead to internalisation of stigma and a sense of hopelessness (Davidson, 2003; Farkas \& Anthony, 2010).

In the last decade, the need to tackle stigma has been included in both global and local mental health policies and research agendas (Saraceno $e t$ $a l, 2007)$. Psychosocial interventions that include peer support work give positive results in reducing stigma among persons with mental illness and professionals (Farkas \& Anthony, 2010). Peer support work is based on the principles of mutuality and hope. It is also based on the belief that persons with mental illness who have gone through difficult situations, and have overcome them, can be 